\title{
The Game-Theoretical Model of Using Insecticide-Treated Bed-Nets to Fight Malaria
}

\author{
Mark Broom ${ }^{1}$, Jan Rychtář ${ }^{2}$, Tracy Spears-Gill ${ }^{3}$ \\ ${ }^{1}$ Department of Mathematics, City University London, Northampton Square, London, UK \\ ${ }^{2}$ Department of Mathematics and Statistics, The University of North Carolina at Greensboro, Greensboro, USA \\ ${ }^{3}$ Department of Biostatistics, University of North Carolina at Chapel Hill, Chapel Hill, USA \\ Email: mark.broom@city.ac.uk,rychtar@uncg.edu,tracygspears@yahoo.com
}

Received 2 April 2016; accepted 23 May 2016; published 26 May 2016

Copyright (C) 2016 by authors and Scientific Research Publishing Inc.

This work is licensed under the Creative Commons Attribution International License (CC BY).

http://creativecommons.org/licenses/by/4.0/

(c) (i) Open Access

\begin{abstract}
Malaria infection is a major problem in many countries. The use of the Insecticide-Treated BedNets (ITNs) has been shown to significantly reduce the number of malaria infections; however, the effectiveness is often jeopardized by improper handling or human behavior such as inconsistent usage. In this paper, we present a game-theoretical model for ITN usage in communities with malaria infections. We show that it is in the individual's self interest to use the ITNs as long as the malaria is present in the community. Such an optimal ITN usage will significantly decrease the malaria prevalence and under some conditions may even lead to complete eradication of the disease.
\end{abstract}

\section{Keywords}

Game Theory, Malaria Prevention, Optimal Strategy, Epidemic Modelling, SIS Model

\section{Introduction}

Malaria is a vector born disease that can be transmitted to people through mosquito bites, prevalent in tropical and subtropical regions of the world. The World Health Organisation estimates that every year nearly one million people die as a result of malaria [1]. A lot of effort has been put into treatment and prevention strategies, and an ambitious goal of organisations such as the World Health Organisation and the Bill and Melinda Gates foundation is the complete elimination of malaria.

One of the key methods of malaria prevention is the use of Insecticide-Treated Bed-Nets (ITNs) that kill and 
also repel mosquitoes [2]. [3] found that the use of ITNs can reduce malaria cases by $50 \%$ and death in children by $20 \%$. The use of ITNs was demonstrated to be very cost-effective, see e.g. [4]-[6]. This is important given both the scale of the problem (about 200 million people are at a very high risk [7]), and the fact that most of the areas of high malaria prevalence are relatively poor. However, human behavior such as inconsistent usage due to hot weather diminishes the effectiveness of the ITNs.

The impact of ITN usage on the spread of the malaria infection has been recently studied in a series of mathematical models such as [8]-[10]. In particular, [10] developed an ODE model for the effects of ITN on the malaria transmission dynamics and called for a better understanding of the impact of human behavior on the usage.

Game theory is a well suited tool to study human behavior in a quantitative way [11]. Starting by [12], game theory has been long used in biology [13], and [14] used it to study vaccination decisions in situation where individuals face a potentially costly preventive action (such as to use an ITN) that can reduce a risk of contracting the disease. Game theory has been applied to vaccination against major public health threats [15]-[21] and it is particularly useful because the outcome of the individual decision (to vaccinate or to use an ITN) depends on the decisions of other members of the population because the overall vaccination or ITN usage yields the disease prevalence in the community [22].

In particular, game theoretical models can be used to understand why in many cases disease eradication can be very hard [14] even when the cost of preventive action is very low [23]. There is often a great incentive to use preventative measures when a disease is at high levels, but when brought below a certain level the incremental benefit of taking the presentative action declines, and so individuals have less incentive to use it. Thus prevention can sometimes (but not always) become ineffective before total eradication occurs. In this paper we adapt a non-game theoretical model of ITN usage [10] to study the impact of individual decisions on the effectiveness of ITN usage.

\section{The Model}

\subsection{Model of Malaria Transmission}

We will use the basic model for the transmission dynamics of malaria infection as presented in [10]. The host population (humans) is divided into two compartments (susceptible and infectious), which are denoted by $S_{h}, I_{h}$, respectively. A total host population is given by $N_{h}=S_{h}+I_{h}$. The vector population (mosquitoes) is also divided into two compartments (susceptible and infectious) which are denoted $S_{v}, I_{v}$, respectively. The total vector population is given by $N_{v}=S_{v}+I_{v}$. When a mosquito bites a human, the susceptible human is infected by an infected mosquito with probability $p_{1}$ and a susceptible mosquito is infected by an infected human with probability $p_{2}$. Hosts die with natural mortality rates being $\mu_{h}$ and can also die because of malaria with rate $\delta_{h}$. Hosts recover with rate $\gamma_{h}$. Vectors can die with rate

$$
\mu_{v}(\bar{b})=\mu_{v_{1}}+\mu_{\max } \bar{b}
$$

where $\mu_{v_{1}}$ is the natural mortality rate of hosts and $\mu_{\max } \bar{b}$ is the mortality rate due to ITN use in the population. Here, $\bar{b} \in[0,1]$ denotes the average ITN usage in the population. Each individual chooses its own individual ITN usage $b \in\{0,1\}$. We assume that all individuals are born as susceptible (with recruitment rates $\Lambda_{h}$ and $\Lambda_{v}$ respectively) and no infected individuals come from outside. All parameters and their values are summarized in Table 1.

Let $\lambda_{h}(b, \bar{b})$ be the force of infection for the focal susceptible host. [10] give the formula

$$
\lambda_{h}(b, \bar{b})=p_{1} \cdot \frac{\beta(b) N_{v}(\bar{b})}{N_{h}(\bar{b})} \cdot \frac{I_{v}(\bar{b})}{N_{v}(\bar{b})}=p_{1} \frac{\beta(b) I_{v}(\bar{b})}{N_{h}(\bar{b})}
$$

where $p_{1}$ is the transmission probability per bite from infectious mosquitoes to humans, $\beta(b)$ is the average number of bites per mosquito per unit of time, $\frac{N_{v}(\bar{b})}{N_{h}(\bar{b})}$ is the average number of mosquitoes per host, and thus $\beta(b) \frac{N_{v}(\bar{b})}{N_{h}(\bar{b})}$ is the average number of bites a susceptible host gets per unit time, and finally $\frac{I_{v}(\bar{b})}{N_{v}(\bar{b})}$ is the 
Table 1. Parameters, their description and value. The cost of malaria infection varies by the country an the used treatment, see for example [28]-[32]. Over the life of the ITN, the total cost of infections is more than the cost of the ITN. Moreover, ITNs are often subsidized, so the actual cost of ITN to the individuals is only around \$1 per ITN [26].

\begin{tabular}{|c|c|c|c|}
\hline & Description & Value & Reference \\
\hline$\mu_{h}$ & Natural mortality for hosts & in $\left[\frac{1}{70 \times 365}, \frac{1}{55 \times 365}\right]$ & {$[10][24]$} \\
\hline$\delta_{h}$ & Diseased induced mortality in hosts & in $\left[0,4.1 \times 10^{-4}\right]$ & [24] \\
\hline$\gamma_{h}$ & Hosts recovery rate & $1 / 14$ & {$[24][25]$} \\
\hline$\mu_{v_{1}}$ & Natural mortality rate for vectors & {$\left[28^{-1}, 21^{-1}\right]$} & {$[10][24]$} \\
\hline$\Lambda_{h}$ & Recruitment rate in hosts & in $\left[10^{3} \mu_{h}, 10^{6} \mu_{h}\right]$ & [8] [10] \\
\hline$\Lambda_{v}$ & Recruitment rate in vectors & in $\left[10^{4} \mu_{v_{1}}, 25000\right]$ & [9] [10] \\
\hline$\beta_{\min }$ & Minimal possible contact rate & 0 & {$[10]$} \\
\hline$\beta_{\max }$ & Maximal possible contact rate & in $[0.9,1]$ & [10] [25] \\
\hline$\mu_{\max }$ & Net related vector mortality rate & $\mu_{v}$ & [10] \\
\hline$p_{1}$ & Prob. of vector to host transmission & 1 & {$[10]$} \\
\hline$p_{2}$ & Prob. of host to vector transmission & 1 & {$[10]$} \\
\hline$C_{M A L}$ & Cost of malaria infection & $>\$ 5$ per case & See caption \\
\hline$C_{\text {ITN }}$ & Cost of net usage & $<\$ 10$ per $3-5$ years & [26] [27] \\
\hline$b$ & Individual ITN usage & in $\{0,1\}$ & \\
\hline $\bar{b}$ & Average ITN usage & in $[0,1]$ & \\
\hline
\end{tabular}

proportion of infectious mosquitoes. [10] considers

$$
\beta(b)=\beta_{\max }-b\left(\beta_{\max }-\beta_{\min }\right)
$$

with $\beta_{\max }=0.1$ and $\beta_{\min }=0$ which yields $\beta(0)=0.1$ and $\beta(1)=0$ (i.e. ITNs provide complete protection).

Also, the average force of the infection is

$$
\lambda_{h}(\bar{b})=p_{1} \frac{\beta(\bar{b}) I_{v}(\bar{b})}{N_{h}(\bar{b})}
$$

and thus

$$
\lambda_{h}(b, \bar{b})=\frac{\beta(b)}{\beta(\bar{b})} \lambda(\bar{b}) .
$$

In the state when malaria reaches equilibrium, [10] evaluates $\lambda_{h}(\bar{b})$ as a solution to

$$
a_{0}\left[\lambda_{h}(\bar{b})\right]^{2}+b_{0}\left[\lambda_{h}(\bar{b})\right]+c_{0}=0,
$$

where

$$
\begin{gathered}
a_{0}=\mu_{v}(\bar{b}) \Lambda_{h}\left(p_{2} \beta(\bar{b})+\mu_{v}(\bar{b})\right) \\
b_{0}=\mu_{v}(\bar{b}) \Lambda_{h} L p_{2} \beta(\bar{b})+2 \mu_{v}^{2}(\bar{b}) \Lambda_{h} L-p_{1} p_{2} \beta^{2}(\bar{b}) \Lambda_{v}\left(\mu_{h}+\delta_{h}\right) \\
c_{0}=\left(\mu_{v}(\bar{b}) L\right)^{2} \Lambda_{h}\left(1-R^{2}\right)
\end{gathered}
$$




$$
\begin{gathered}
L=\mu_{h}+\gamma_{h}+\delta_{h} \\
R^{2}=\frac{\beta^{2}(\bar{b})}{\mu_{v}^{2}(\bar{b})} \cdot \frac{p_{1} p_{2} \Lambda_{v} \mu_{h}}{L \Lambda_{h}}
\end{gathered}
$$

In [10] it is shown that there are potentially two stable steady states of infections and if the malaria was originally common, the stable states yields

$$
\lambda_{h}(\bar{b})= \begin{cases}\frac{-b_{0}+\sqrt{b_{0}^{2}-4 a_{0} c_{0}}}{2 a_{0}}, & \text { if } b_{0}^{2}-4 a_{0} c_{0}>0 \\ 0 ; & \text { otherwise. }\end{cases}
$$

Figure 1 shows values of $\lambda_{h}(\bar{b})$ for specific parameter values. Note that $\lambda_{h}(\bar{b})$ eventually becomes 0 (quite abruptly as seen in Figure 1 (a)) as $\bar{b}$ increases. This is caused by the fact that for large $\bar{b}$, the disease can be eradicated according to the model presented in [10].

\subsection{Game-Theoretical Model}

Let $E(b, \bar{b})$ be the payoff for a focal individual using $(b=1)$ or not using $(b=0)$ the ITN in a population where the average use of ITNs is $\bar{b} \in[0,1]$. We shall find the ITN usage equilibrium usage level $\bar{b}_{s}$ satisfying

$$
E(1, \bar{b})-E(0, \bar{b}) \lessgtr 0 \text { if and only if } \bar{b}_{s} \lessgtr \bar{b} \text {. }
$$

Such a usage level is stable, because if $\bar{b}>\bar{b}_{s}$, then $E(1, \bar{b})<E(0, \bar{b})$ and thus individuals are better off not using ITNs, and the average population usage level will thus go down (provided the individuals acts rationally). On the other hand, if $\bar{b}<\bar{b}_{s}$, then $E(1, \bar{b})>E(0, \bar{b})$ and thus individuals are better off if they use the ITN and the population usage will thus go up. Here we assume that while individuals cannot accurately assess the overall state of the population (in particular the prevalence of malaria) instantaneously, they can change their behavior at a faster rate than the state of the population changes, in particular when the disease prevalence is either very high or very low. This seems reasonable, since individuals have a complete control over whether they use an ITN and can adapt quickly (though how they act will depend upon the information that they have available, which we consider in the Discussion).

Following [14], we will evaluate

$$
E(b, \bar{b})=-c b-\pi(b, \bar{b})
$$

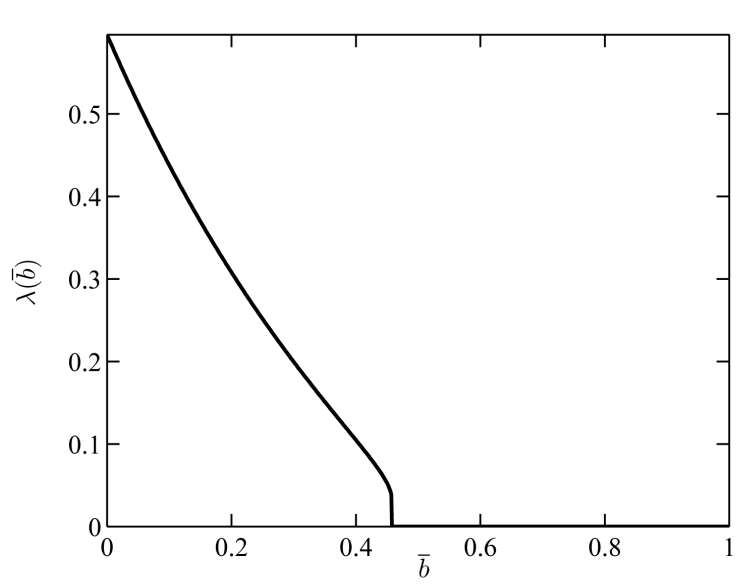

(a)

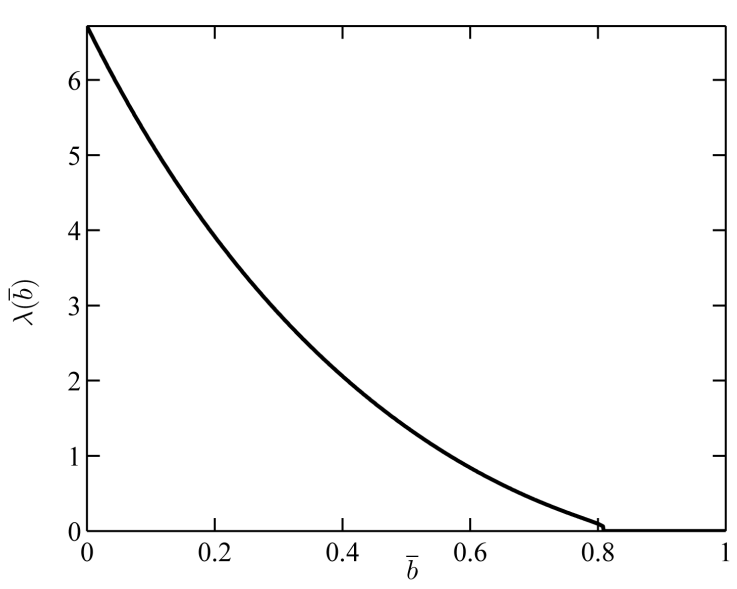

(b)

Figure 1. Values of $\lambda(\bar{b})$ for different vector recruitment rates. (a) $\Lambda_{v}=10^{3} \mu_{v_{1}}$, (b) $\Lambda_{v}=10^{4} \mu_{v_{1}}$. Values of the remaining parameters are $\mu_{v_{1}}=1 / 28, \mu_{\max }=\mu_{v_{1}}, \beta_{\max }=0.1, \beta_{\min }=0, \mu_{h}=1 /(55 \times 365), p_{1}=1, p_{2}=1, \Lambda_{h}=10^{3} \mu_{h}, \delta_{h}=4.1 \times 10^{-4}, \gamma_{h}=1 / 14$. 
where $\pi(b, \bar{b})$ is the probability of the focal individual getting infected in a population with average ITN usage $\bar{b}$ and $c=c_{I T N} / c_{M A L}$ is the relative cost of the ITN and the cost of getting infected by malaria.

To evaluate $\pi(b, \bar{b})$, we will use and adapt the model developed in [10]. The probability of a host getting infected is then given by the ratio of the rate of getting infected to the rate of leaving the susceptible state for any reason. Thus,

$$
\pi(b, \bar{b})=\frac{\lambda_{h}(b, \bar{b})}{\lambda_{h}(b, \bar{b})+\mu_{h}} .
$$

\section{Analysis}

Given a relatively small value of $\mu_{h}$ and a relatively large value of $\lambda_{h}(\bar{b})$, we get from (15) and (5)

$$
\pi(0, \bar{b})\left\{\begin{array}{cc}
\approx 1-\frac{\beta(\bar{b})}{\beta_{\max }} \frac{\mu_{h}}{\lambda_{h}(\bar{b})}, & \text { if } \lambda_{h}(\bar{b})>0, \\
=0, & \text { if } \lambda_{h}(\bar{b})=0, \\
\pi(1, \bar{b})=0 . &
\end{array}\right.
$$

There will of course be values of $\lambda_{h}(\bar{b})$ just above 0 which are not small compared to $\mu_{h}$, but for the parameters in our model (see Table 1) this happens for a very narrow range of values of $\bar{b}$, and so the above is a good approximation.

It follows from (14) that

$$
\begin{gathered}
E(1, \bar{b})=-c-\pi(1, \bar{b})=-c \\
E(0, \bar{b})=0-\pi(0, \bar{b}),
\end{gathered}
$$

and thus, by (16), (17),

$$
E(1, \bar{b})-E(0, \bar{b})=\pi(0, \bar{b})-c= \begin{cases}\pi(0, \bar{b})-c & \text { if } \lambda_{h}(\bar{b})>0, \\ -c, & \text { if } \lambda_{h}(\bar{b})=0 .\end{cases}
$$

Because by (16), $\pi(0, \bar{b}) \approx 1$ for most $\bar{b}$ such that $\lambda_{h}(\bar{b})>0$, we get that

$$
E(1, \bar{b})-E(0, \bar{b})<0 \text {, for all } \bar{b} \in[0,1] \text { if } c=c_{\text {ITN }} / c_{\text {MAL }}>1
$$

and otherwise there is a unique $\bar{b}_{s}$ satisfying the equilibrium condition (13). The condition $c>1$ is however not realistic for real populations, and thus in practice we will always get a unique $\bar{b}_{s}$. The plots of $E(1, \bar{b})-E(0, \bar{b})$ are shown in Figure 2.

Moreover, the unique $\bar{b}_{s}$ is given exactly as a solution of

$$
b_{0}^{2}-4 a_{0} c_{0}=0
$$

where $a_{0}, b_{0}$ and $c_{0}$ are given by (7)-(9). As in [10], (22) happens for a critical value $R_{c}$ given by

$$
R_{c}=\sqrt{1-\frac{b_{0}^{2}}{4 a_{0} \Lambda_{h}\left(\mu_{v} L\right)^{2}}}
$$

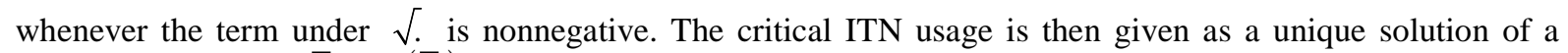
fixed point problem $\bar{b}_{s}=b_{c}\left(\bar{b}_{s}\right)$ where

$$
b_{c}(\bar{b})= \begin{cases}\frac{\beta_{\max }-\mu_{v_{1}} Q R_{c}}{\beta_{\max }-\beta_{\min }+Q R_{c} \mu_{\max }}, & \text { if } R_{c} \text { is defined, } \\ 1, & \text { otherwise, }\end{cases}
$$

where $Q=\sqrt{L \Lambda_{h} / \Lambda_{v} \mu_{h} p_{1} p_{2}}$. The plots of $b_{c}(\bar{b})$ for different parameter values are shown at Figure 3 . 


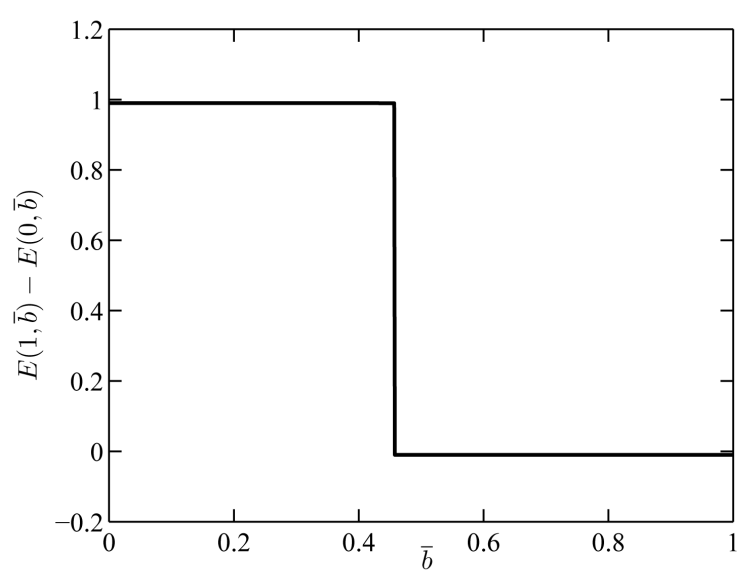

(a)

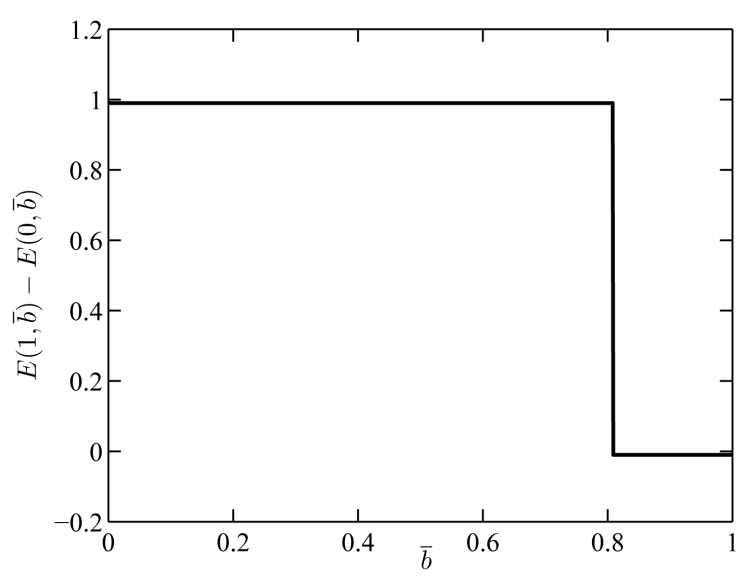

(b)

Figure 2. Values of $E(1, \bar{b})-E(0, \bar{b})$ for different vector recruitment rates. (a) $\Lambda_{v}=10^{3} \mu_{v_{1}}$, (b) $\Lambda_{v}=10^{4} \mu_{v_{1}}$. Values of the remaining parameters are $\mu_{v_{1}}=1 / 28, \mu_{\max }=\mu_{v_{1}}, \beta_{\max }=0.1, \beta_{\min }=0, \mu_{h}=1 /(55 \times 365), p_{1}=1, p_{2}=1, \Lambda_{h}=10^{3} \mu_{h}, \delta_{h}=4.1 \times$ $10^{-4}, \gamma_{h}=1 / 14$

\section{Results}

It follows from our analysis that it is in the individual's best interest to use the ITN as long as there is malaria present in the population. For the parameter values used above, namely $\beta_{\min }=0$, the optimal usage (from the individual's perspective) coincide with the average usage in the population that eradicates the disease.

However, the model used shows particular sensitivity to the parameter value $\beta_{\min }$. This is unfortunate because the effectiveness of the insecticide sprayed on the ITN deteriorates over 3 - 5 years [2], the net cannot effectively provide protection for the whole 24 hours, and the mosquitoes may adapt their biting behavior in areas of high ITN coverage [33]. Consequently, the realistic value of $\beta_{\min }$ is positive and can be relatively close to $\beta_{\max }$. As shown in Figure 4 when $\beta_{\min }=0.075$ (or even $\beta_{\min }=0.025$ for larger values of $\Lambda_{v}$ ), the malaria does not get eradicated. Still, the force of infection, $\lambda_{h}(\bar{b})$, decreases with $\bar{b}$; and the decrease is significant. The quantity $E(1, \bar{b})-E(0, \bar{b})$ is also always positive (close to 1 ), thus it is in the individual's interest to keep using the ITN. Consequently, the ITN usage does not eradicate the disease, but helps to significantly reduce its force.

However, in all of the above, we considered that malaria was transmitted between vectors and host during every bite. If we relax this assumption and consider $p_{1}, p_{2}<1$, then again malaria can be eradicated even for larger values of $\beta_{\min }$ as in Figure 4(c) and Figure 4(d).

\section{Discussion}

In this paper we have considered a game-theoretical model of ITN usage, allowing individuals in the population to decide whether (and how often) they use ITNs. We have shown that optimal behavior from the individual's perspective leads to the decrease (or even eradication) of the disease, i.e. an optimal outcome from the population perspective. In particular if it is not possible to eradicate the disease through ITN usage, individuals use ITNs all the time, which leads to the minimum possible level of malaria. If eradication is possible, individuals select a strategy which is approximately at the level that leads to eradication. Moreover, the optimal behavior does not depend on the exact cost of the ITN use (as long as the cost is smaller than the cost of the disease). This type of outcome is not always the case; see for example [14].

We have seen that individual strategies lead to the eradication level effectively independently of the cost in our case, because individuals have a very high likelihood of getting infected by the disease whenever the disease is present in the population, due to the high rate of infection $\left(\lambda_{h}\right)$, which is much higher than the rate of natural death, meaning that people become infected with probability almost equal to 1 .

We have assumed that individuals are able to adjust their ITN usage strategy faster than changes in the underlying state of the population, such as the prevalence of the disease, to always obtain the optimal level of 


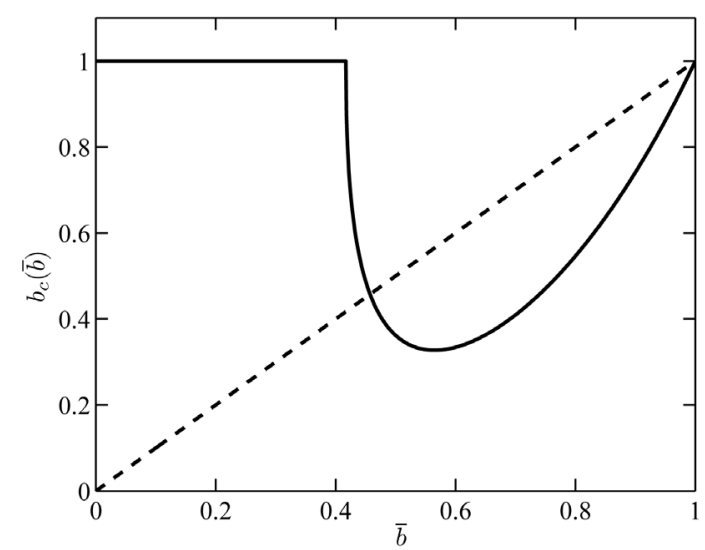

(a)

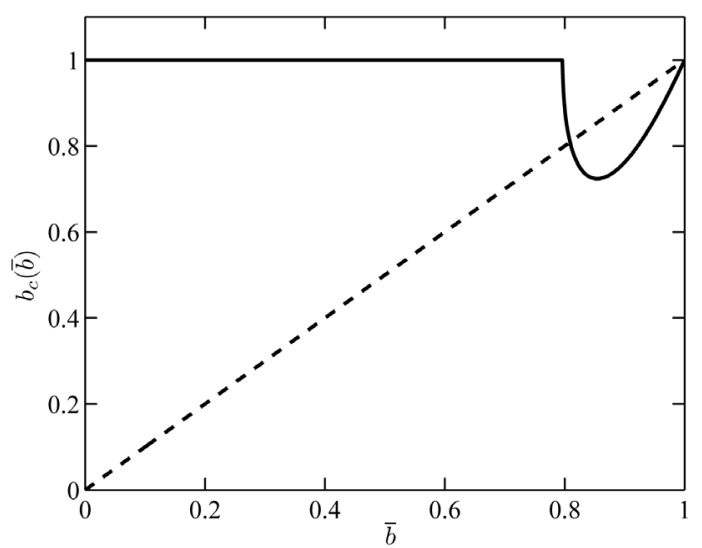

(b)

Figure 3. Plots of $b_{c}(\bar{b})$ for different vector recruitment rates. (a) $\Lambda_{v}=10^{3} \mu_{v_{1}}$, (b) $\Lambda_{v}=10^{4} \mu_{v_{1}}$. Values of the remaining parameters are $\mu_{v_{1}}=1 / 28, \mu_{\max }=\mu_{v_{1}}, \beta_{\max }=0.1, \beta_{\min }=0, \mu_{h}=1 /(55 \times 365), p_{1}=1, p_{2}=1, \Lambda_{h}=10^{3} \mu_{h}, \delta_{h}=4.1 \times 10^{-4}, \gamma_{h}=1 / 14$.

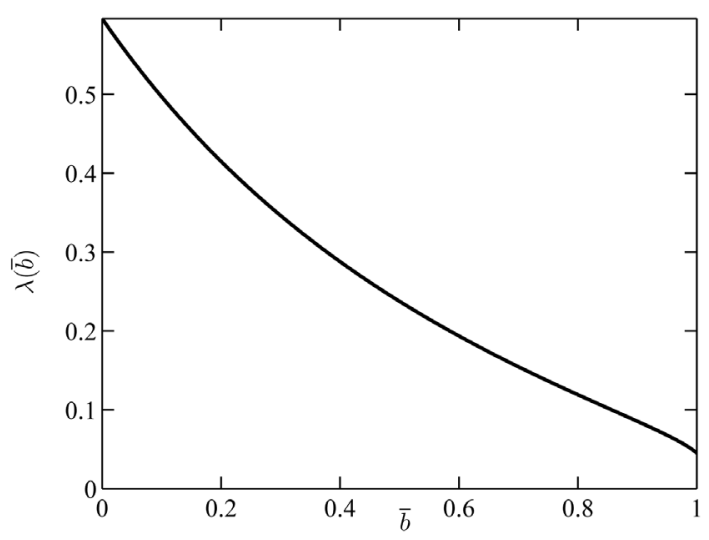

(a)

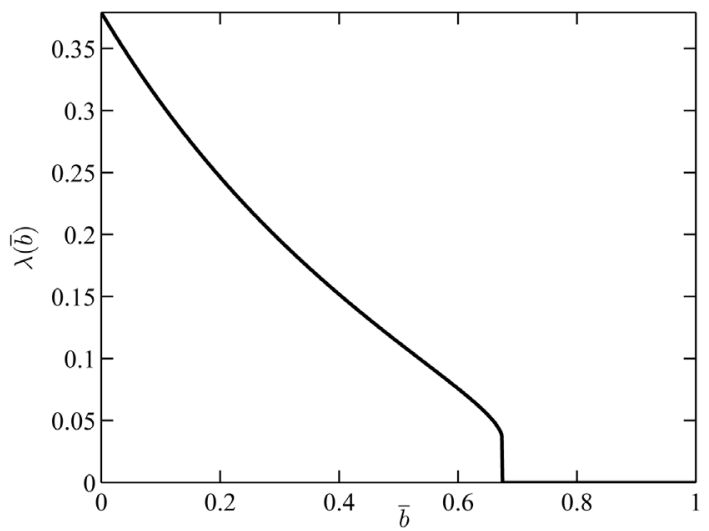

(c)

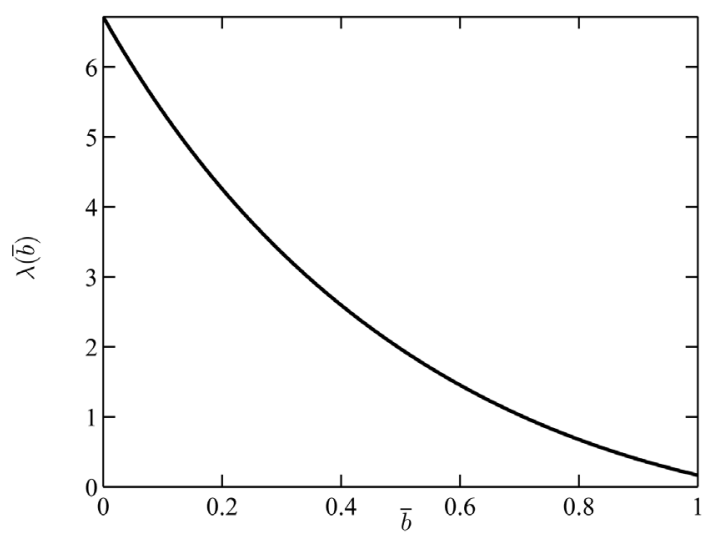

(b)

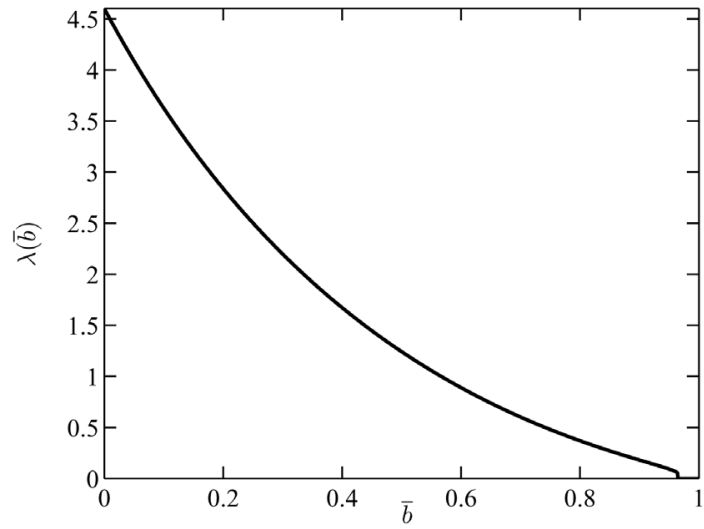

(d)

Figure 4. Values of $\lambda(\bar{b})$ for different vector recruitment rates and minimal contact rates. (a) $\Lambda_{v}=10^{3} \mu_{v_{1}}$, $\beta_{\text {min }}=0.075, p_{1}=1, p_{2}=1$, (b) $\Lambda_{v}=10^{4} \mu_{v_{1}}, \beta_{\text {min }}=0.025, p_{1}=1, p_{2}=1$, (c) $\Lambda_{v}=10^{3} \mu_{v_{1}}, \beta_{\min }=0.075, p_{1}=0.75, p_{2}=0.75$ ， (d) $\Lambda_{v}=10^{4} \mu_{v_{1}}, \beta_{\min }=0.025, p_{1}=0.75, p_{2}=0.75$. Values of the remaining parameters are $\mu_{v_{1}}=1 / 28, \mu_{\max }=\mu_{v_{1}}, \beta_{\max }=0.1$, $\mu_{h}=1 /(55 \times 365), \Lambda_{h}=10^{3} \mu_{h}, \delta_{h}=4.1 \times 10^{-4}, \gamma_{h}=1 / 14$. 
usage. It is clear that they are physically able to do this, because to put up or remove an ITN takes a relatively short time. However, they clearly need sufficient information about the state of the population to make a rational decision to change. What information would they have on which to base their decision? It is clear from Figure 2 that except for $\bar{b}$ within a very narrow range the disease is either absent or effectively everyone has it; this information is obvious, and so any individual with the wrong strategy would quickly change it. Thus the population usage would quickly evolve to a level close to the critical level that we have assumed.

We note that because of the high infection rate, a level of ITN usage close to the eradication level but still below it could formally still lead to high levels of disease prevalence. Thus if individuals had perfect information about the state of the population they could choose their strategies to achieve the precise level of the equilibrium strategy, which would lead to some intermediate level of infection between 0 and 1 . It is our assumption in this paper that they cannot do this precisely. In any case natural variations within the real population parameters, would mean that the exact value of this critical level would be subject to some fluctuation. The consequence is that the chosen strategy is sufficiently close to the eradication level, that there is a good chance of such eradication occurring, on the assumption that individuals play a fixed bed-net usage strategy.

In reality, it may be that the level of malaria will not have enough time to tend to 0 before individuals in the population notice the low level and give up the use of ITNs. Thus if the malaria level is significantly different to the equilibrium level from our analysis, individual behaviour will prevent it from being eliminated. Thus a more complex dynamically coupled model of the evolution of ITN usage and malaria level may be needed to decide the precise behaviour at such intermediate levels of infection.

\section{Acknowledgements}

The research was supported by the Simons Foundation grant \#245400 (Jan Rychtář).

\section{References}

[1] WHO (2005) World Malaria Report.

[2] Raghavendra, K., Barik, T.K., Niranjan Reddy, B.P., Sharma, P. and Dash, A.P. (2011) Malaria Vector Control: From Past to Future. Parasitology Research, 108, 757-779. http://dx.doi.org/10.1007/s00436-010-2232-0

[3] Lengeler, C. (2004) Insecticide-Treated Bed Nets and Curtains for Preventing Malaria. Cochrane Database of Systematic Reviews, 2, Article ID: CD000363. http://dx.doi.org/10.1002/14651858.cd000363.pub2

[4] Goodman, C.A. and Mills, A.J. (1999) The Evidence Base on the Cost-Effectiveness of Malaria Control Measures in Africa. Health Policy and Planning, 14, 301-312. http://dx.doi.org/10.1093/heapol/14.4.301

[5] Goodman, C.A., Coleman, P.G. and Mills, A.J. (1999) Cost-Effectiveness of Malaria Control in Sub-Saharan Africa. The Lancet, 354, 378-385. http://dx.doi.org/10.1016/S0140-6736(99)02141-8

[6] White, M.T., Conteh, L., Cibulskis, R. and Ghani, A.C. (2011) Costs and Cost-Effectiveness of Malaria Control Interventions-A Systematic Review. Malaria Journal, 10, 1475-2875. http://dx.doi.org/10.1186/1475-2875-10-337

[7] Miller, J.M., Korenromp, E.L., Nahlen, B.L. and Steketee, R.W. (2007) Estimating the Number of Insecticide-Treated Nets Required by African Households to Reach Continent-Wide Malaria Coverage Targets. JAMA, 297, 2241-2250. http://dx.doi.org/10.1001/jama.297.20.2241

[8] White, L.J., Maude, R.J., Pongtavornpinyo, W., Saralamba, S., Aguas, R., Van Effelterre, T., Day, N.P.J. and White, N.J. (2009) The Role of Simple Mathematical Models in Malaria Elimination Strategy Design. Malaria Journal, 8, 212. http://dx.doi.org/10.1186/1475-2875-8-212

[9] Chitnis, N., Schapira, A., Smith, T. and Steketee, R. (2010) Comparing the Effectiveness of Malaria Vector-Control Interventions through a Mathematical Model. The American Journal of Tropical Medicine and Hygiene, 83, 230. http://dx.doi.org/10.4269/ajtmh.2010.09-0179

[10] Agusto, F.B., Del Valle, S.Y., Blayneh, K.W., Ngonghala, C.N., Goncalves, M.J., Li, N.P., Zhao, R.J. and Gong, H.F. (2013) The Impact of Bed-Net Use on Malaria Prevalence. Journal of Theoretical Biology, 320, 58-65. http://dx.doi.org/10.1016/j.jtbi.2012.12.007

[11] Von Neumann, J. and Morgenstern, O. (2007) Theory of Games and Economic Behavior (Commemorative Edition). Princeton University Press.

[12] Smith, J.M. and Price, G.R. (1973) The Logic of Animal Conflict. Nature, 246, 15-18. http://dx.doi.org/10.1038/246015a0

[13] Broom, M. and Rychtáŕ, J. (2013) Game-Theoretical Models in Biology. CRC Press. 
[14] Bauch, C.T. and Earn, D.J.D. (2004) Vaccination and the Theory of Games. Proceedings of the National Academy of Sciences of the United States of America, 101, 13391-13394. http://dx.doi.org/10.1073/pnas.0403823101

[15] Bauch, C.T., Galvani, A.P. and Earn, D.J.D. (2003) Group Interest versus Self-Interest in Smallpox Vaccination Policy. Proceedings of the National Academy of Sciences of the United States of America, 100, 10564-10567. http://dx.doi.org/10.1073/pnas.1731324100

[16] Bauch, C.T. (2005) Imitation Dynamics Predict Vaccinating Behaviour. Proceedings of the Royal Society B: Biological Sciences, 272, 1669-1675. http://dx.doi.org/10.1098/rspb.2005.3153

[17] Galvani, A.P., Reluga, T.C. and Chapman, G.B. (2007) Long-Standing Influenza Vaccination Policy Is in Accord with Individual Self-Interest but Not with the Utilitarian Optimum. Proceedings of the National Academy of Sciences, 104, 5692-5697. http://dx.doi.org/10.1073/pnas.0606774104

[18] Shim, E., Kochin, B. and Galvani, A. (2009) Insights from Epidemiological Game Theory into Gender-Specific Vaccination against Rubella. Mathematical Biosciences and Engineering: MBE, 6, 839-854. http://dx.doi.org/10.3934/mbe.2009.6.839

[19] Shim, E., Grefenstette, J.J., Albert, S.M., Cakouros, B.E. and Burke, D.S. (2012) A Game Dynamic Model for Vaccine Skeptics and Vaccine Believers: Measles as an Example. Journal of Theoretical Biology, 295, 194-203. http://dx.doi.org/10.1016/j.jtbi.2011.11.005

[20] Crawford, K., Lancaster, A., Oh, H. and Rychtář, J. (2015) A Voluntary Use of Insecticide-Treated Cattle Can Eliminate African Sleeping Sickness. Letters in Biomathematics, 2, 91-101. http://dx.doi.org/10.1080/23737867.2015.1111777

[21] Sykes, D. and Rychtář, J. (2015) A Game-Theoretic Approach to Valuating Toxoplasmosis Vaccination Strategies. Theoretical Population Biology, 105, 33-38. http://dx.doi.org/10.1016/j.tpb.2015.08.003

[22] Shim, E., Chapman, G.B., Townsend, J.P. and Galvani, A.P. (2012) The Influence of Altruism on Influenza Vaccination Decisions. Journal of the Royal Society Interface, 9, 2234-2243.

[23] Geoffard, P.-Y. and Philipson, T. (1997) Disease Eradication: Private versus Public Vaccination. The American Economic Review, 87, 222-230.

[24] Teboh-Ewungkem, M.I., Podder, C.N. and Gumel, A.B. (2010) Mathematical Study of the Role of Gametocytes and an Imperfect Vaccine on Malaria Transmission Dynamics. Bulletin of Mathematical Biology, 72, 63-93. http://dx.doi.org/10.1007/s11538-009-9437-3

[25] Bowman, C., Gumel, A.B., van den Driessche, P., Wu, J. and Zhu, H. (2005) A Mathematical Model for Assessing Control Strategies against West Nile Virus. Bulletin of Mathematical Biology, 67, 1107-1133. http://dx.doi.org/10.1016/j.bulm.2005.01.002

[26] Guyatt, H.L., Kinnear, J., Burini, M. and Snow, R.W. (2002) A Comparative Cost Analysis of Insecticide-Treated Nets and Indoor Residual Spraying in Highland Kenya. Health Policy and Planning, 17, 144-153. http://dx.doi.org/10.1093/heapol/17.2.144

[27] Mulligan, J.-A., Yukich, J. and Hanson, K. (2008) Costs and Effects of the Tanzanian National Voucher Scheme for Insecticide-Treated Nets. Malaria Journal, 7, 32. http://dx.doi.org/10.1186/1475-2875-7-32

[28] Asenso-Okyere, W.K. and Dzator, J.A. (1997) Household Cost of Seeking Malaria Care. A Retrospective Study of Two Districts in Ghana. Social Science \& Medicine, 45, 659-667. http://dx.doi.org/10.1016/S0277-9536(96)00383-8

[29] Akazili, J., Aikins, M. and Binka, F.N. (2008) Malaria Treatment in Northern Ghana: What Is the Treatment Cost per Case to Households? African Journal of Health Sciences, 14, 70-79. http://dx.doi.org/10.4314/ajhs.v14i1.30849

[30] Mutabingwa, T.K. (2005) Artemisinin-Based Combination Therapies (ACTs): Best Hope for Malaria Treatment but Inaccessible to the Needy! Acta Tropica, 95, 305-315. http://dx.doi.org/10.1016/j.actatropica.2005.06.009

[31] Whitty, C.J.M., Chandler, C., Ansah, E., Leslie, T. and Staedke, S.G. (2008) Deployment of ACT Antimalarials for Treatment of Malaria: Challenges and Opportunities. Malaria Journal, 7, S7. http://dx.doi.org/10.1186/1475-2875-7-S1-S7

[32] Lubell, Y., Reyburn, H., Mbakilwa, H., Mwangi, R., Chonya, S., Whitty, C.J.M. and Mills, A. (2008) The Impact of Response to the Results of Diagnostic Tests for Malaria: Cost-Benefit Analysis. BMJ, 336, 202-205. http://dx.doi.org/10.1136/bmj.39395.696065.47

[33] Killeen, G.F., Kihonda, J., Lyimo, E., Oketch, F.R., Kotas, M.E., Mathenge, E., Schellenberg, J.A., Lengeler, C., Smith, T.A. and Drakeley, C.J. (2006) Quantifying Behavioural Interactions between Humans and Mosquitoes: Evaluating the Protective Efficacy of Insecticidal Nets against Malaria Transmission in Rural Tanzania. BMC Infectious Diseases, 6, 161. http://dx.doi.org/10.1186/1471-2334-6-161 